\title{
Rare and Hereditary Causes of Stroke-A Literature Review
}

\section{Eyisi $\mathrm{CS}^{1 *}$, Onwuekwe $I \mathrm{O}^{1}$, Eyisi I $\mathrm{G}^{2}$ and Ekenze $\mathrm{O}^{1}$}

\author{
${ }^{1}$ Neurology Unit, Department of Medicine, College of Medicine, University of Nigeria Teaching Hospital, \\ Nigeria \\ 2Department of Community Medicine, College of Medicine, Chukwuemeka Odumegwu Ojukwu University, \\ Nigeria
}

*Corresponding author: Dr. Eyisi Chioma, Neurology Unit, Department of Medicine, University of Nigeria Teaching Hospital, Ituku-Ozalla, Enugu State, Nigeria, Tel: +2348168858498

\begin{abstract}
Background: Rare and hereditary diseases are important in causing stroke in children, younger patients and women [1]. These diseases have a world-wide prevalence, however they are not commonly diagnosed in Nigeria because of unavailability of gene mapping technologies. Notable exceptions include Moyamoya disease which commonly occur in Asian populations and Chronic Myelogenous Leukemia which causes stroke in the $7^{\text {th }}$ and $8^{\text {th }}$ decade, in contrast to other diseases that cause stroke in the $1^{\text {st }}$ to $3^{\text {rd }}$ decades [1].
\end{abstract}

Aim: The aim of this paper is to review several rare and hereditary causes of stroke.

Methodology: A literature search of various database.

Results: Stroke in young people have been linked to causes other than the conventional risk factors. In Africa, Sickle Cell Anemia is a leading cause [1]. International research consortium (MEGASTROKE) studying 520,000 individuals from around the world have identified about 22 new genetic risk factors for stroke [2]

There are currently about 32 independent genomic regions associated with stroke [2]. Other influences including hereditary causes have been associated with about $50 \%$ of strokes [2].

\section{Keywords}

Stroke, Hereditary, Africa

\section{Introduction}

A stroke is a Focal (or global) neurological impairment of sudden onset, lasting more than $24 \mathrm{hrs}$ and usually from a vascular cause [1]. Clinical presentation span through a myriad of motor deficits, sensory deficits, seizures, personality/behavioral anomalies, dementia, brainstem stroke syndromes and herniation syndromes.

Traditionally stokes are classified as ischemic or hemorrhagic, the former more common than the latter in a ratio of $6: 1$ [1]. Conventional risk factors for stroke occurrence have also be described and grouped into modifiable and non-modifiable risk factors [1]. Modifiable risk factors include hypertension, Smoking, Lifestyle, Alcohol, High cholesterol, Atrial fibrillation, Obesity, Diabetes, Severe carotid stenosis, Sleep apnea. Non-modifiable risk factors comprise of Male sex, African race and age of more than 40 years [1].

Single gene disorders have been well studied in relation to stroke [3]. Diseases under this category include CADASIL (cerebral autosomal dominant arteriopathy with subcortical infarcts and leukoencephalopathy), CARASIL (cerebral autosomal recessive arteriopathy with subcortical infarcts and leukoencephalopathy), Fabry's disease and Sickle Cell Disease. On the other hand, the study of polygenic diseases causing stroke is somewhat cumbersome due to gene polymorphisms. A study done by Framingham with the aim of investigating the familial aggregation of stroke in different lacunar subtype strokes, showed that parental occurrence of stroke by 65 years increased the risk of ischemic stroke in the offspring by 3 -fold and this persisted after adjustment of conventional stroke risk factors [4]. Also, several studies have linked Phosphodiesterase 4D with multifactorial strokes [5]. Phosphodiesterase 4D is among the class of enzymes that selectively degrades cAMP, a signal transduction protein. In vascular smooth muscle, low cAMP leads to increased cell proliferation.

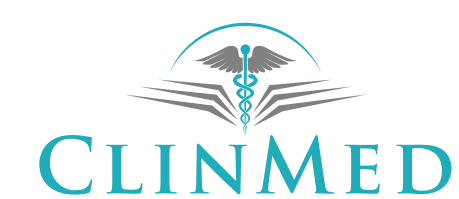

INTERNATIONAL LIBRARY

Citation: Eyisi CS, Onwuekwe IO, Eyisi IG, Ekenze O (2018) Rare and Hereditary Causes of Stroke-A Literature Review. Int J Neurodegener Dis 1:005

Accepted: November 17, 2018; Published: November 19, 2018

Copyright: (c) 2018 Eyisi CS, et al. This is an open-access article distributed under the terms of the Creative Commons Attribution License, which permits unrestricted use, distribution, and reproduction in any medium, provided the original author and source are credited. 
Phosphodiesterase 4D has also been linked with asthma and myocardial infarction [5].

\section{Causes and Pathophysiological Mechanisms}

These diseases basically involve a thrombophilic state (less commonly a bleeding tendency state) which is the same underlying mechanism for strokes. Virchow's triad (hypercoagulability, stasis \& endothelial dysfunction) is an important cycle in propagating thrombophilia. For the purposes of this paper, these diseases have been classified into:

- Hematological Disorders e.g. Hypercoagulable states.

- Inflammatory Blood Vessel Disorders e.g. Vasculitis.

- Non-Inflammatory Blood Vessel Disorder.

- Metabolic Disorders e.g. Fabry's disease.

- Drugs e.g. Cocaine, Oral Contraceptive Pills.

- Miscellaneous e.g. Infective endocarditis, Congenital Heart Diseases.

\section{Hematologic Disorders}

\section{Sickle Cell Anemia (SCD)}

A Monogenic disorder (AR), due to point mutation on chromosome 11, with change of glutamate to valine, affects b-subunit of globin chain. It is inherited in a homozygous pattern and commonly causes stroke in children. Result of this mutation is an altered erythrocyte shape and the mechanism of causing stroke could be vaso-occlusive or anaemic where it affects the carotids and MCA commonly. Misshapen red cells are non-deformable in the $0.3 \mathrm{~nm}$ capillary lumen leading to clogging of blood vessels especially under hypoxic conditions (acidosis, infections, dehydration etc). MCA Doppler predicts occurrence of stroke in SCD children (high velocity flow increases risk of stroke) [6].

\section{Anti-phospholipid Antibody Syndrome (APS)}

An autoimmune disease, common in females around 15-55 years. It is usually associated with other autoimmune diseases. A familial disease and possibly a polygenic disorder. It is characterized by antibodies against phospholipids on cell membranes (aPLs) which can be elaborated during infectious processes (hepatitis, HIV, TB, Lyme's dix). Antibodies also usurp endothelial PGE2/ TXA2, enhances platelets activity and dysregulates complement system leading to increased thrombosis. Arterial and venous thrombosis can occur, also obstetric features are present. Obstetric clinical diagnostic criteria ( 3 consecutive abortions) are available [4].

\section{Polycythemia Rubra Vera (PRV)}

A neoplastic proliferation of erythroids (with or without other cell lines). It has no sex predilection and can occur in childhood or adulthood. A Monogenic disorder with point mutation (V617F) on JAK2STAT kinase gene which results in permanent growth signals in the nucleus of erythroid precursors. Mutations on exon 12 also lead to PRV. It is associated with thrombosis due to polycythemia and bleeding episodes as a result of disrupted hemostatic mechanisms. Diagnostic criteria available [4].

\section{Essential Thrombocytosis (ET)}

A myeloproliferative disorder characterized by autonomous platelet production, decreased effect of platelet inhibiting factor and defects in hematologic microenvironment. JAK2 mutations and MPL (Myeloproliferative leukemia virus oncogene) mutations have been described. It causes a sustained platelet production leading to thrombomegalia and eventually a vascular thrombosis occurs.

\section{Chronic Myelogenous Leukemia (CML)}

A disorder characterized by an increased proliferation of mature granulocytes. Genetic mutation implicated is the BCR/ABL fusion, popularly called Philadelphia chromosome. High blood levels of granulocytes lead to hyperviscosity, leucostasis and thrombosis.

\section{Factor V Leiden}

An autosomal dominant disease with incomplete penetrance due to a missense substitution of Guanine to Adenine and protein change from arginine to glutamine. Factor $V$ variant produced cannot be degraded by activated Protein $\mathrm{C}$. this predisposes to a hypercoagulable state and thrombosis. Venous thrombosis (DVTs) are commoner but arterial thrombosis and strokes can occur.

\section{Protein C deficiency}

Protein $\mathrm{C}$ normally inactivates pro-coagulant factors Va and VIIla. It is in turn activated by a thrombin-thrombomodulin complex (found on normal endothelium) so it delimits the site where blood clotting occurs. Deficien$c y$ is inherited in $A D$ fashion and various mutations have been implicated with chromosome 2 mutations more popularly studied. It can also be acquired in patients with severe liver disease, biliary disease or Vitamin K deficiency. Protein $\mathrm{C}$ deficiency can be qualitative or quantitative. This disorder leads to thrombophilia resulting in venous and arterial thrombosis.

\section{Protein S deficiency}

Protein $\mathrm{S}$ is a cofactor for Protein C. Deficiency can be heritable or acquired and mutation involves several genes. It leads to thrombophilia.

Inflammatory Blood Vessel Disorders (Vasculitis)

\section{Giant cell arteritis}

A large vessel vasculitis more common with female predilection. It is characterized by inflammation of external carotid artery branches (temporal arteries); gran- 
ulomatous inflammation with typical giant cells on histology. It rarely causes stroke because internal carotid artery is not affected but it can lead to blindness due to involvement of ophthalmic arteries.

\section{Takayasu arteritis (Pulseless disease)}

A large vessel granulomatous vasculitis, common in younger women. It involves inflammation of aorta and its branches, also pulmonary vessels are affected and it causes carotid or vertebral artery thrombosis.

\section{Polyarteritis nodosa}

A medium vessel vasculitis especially occurring at points of bifurcations which results in micro-aneurysms, vessel rupture and hemorrhage. Also, thrombosis can occur in inflamed parts of the vessel wall. It commonly affects coronary vessels, renal vessels and cerebral vessels.

\section{Wegener's granulomatosis}

A small vessel vasculitis which affects small branches $(<2 \mathrm{~mm})$ of the main intra-cerebral vessels. This leads to small infarcts in CNS and eyes.

\section{Isolated angitis of the CNS}

A small and medium vessel vasculitis primarily in CNS. No systemic vasculitis occurs. The mechanism of causing stroke is commonly ischemic.

\section{Vasculitis in other autoimmune diseases}

Antibodies produced in autoimmune diseases like SLE can damage blood vessel wall and causes ischemic stroke if cerebral vessels are affected. Also, Libman-sacks endocarditis in patients with SLE can cause cardio-embolic stroke.

\section{Vasculitis in infectious diseases}

Human Immunodeficiency Virus infection accounts for about $7 \%$ of cases of young stroke in Nigeria [7]. Vasculitis is proposed to be the underlying mechanism.

\section{Non-Inflammatory Blood Vessel Disorder}

\section{Fibromuscular dysplasia}

A progressive narrowing of blood vessels throughout the body. It affects medium size vessels. The etiology unknown but an underlying connective tissue disorder is implicated. It occurs commonly in women. It is characterized by multiple rings of segmental narrowing alternating with dilatation of carotid and vertebral vessels. It can cause a TIA or stroke.

\section{Moyamoya disease}

A vaso-occlusive disease of large intracranial vessels (ICA, stem of ACA and MCA, Circle of willis). It is common in females. The lenticulostriate arteries develop a rich collateral circulation around the occlusive lesion, which gives the impression of a "puff of smoke" (moy- amoya in Japanese) on conventional $\mathrm{x}$-ray angiography. A progressive condition with unknown etiology.

\section{CADASIL (Cerebral Autosomal Dominant Arteriop- athy with Subcortical Infarcts and Leukoencepha- lopathy)}

It is inherited in AD fashion and occurs due to mutations in Notch3 (EGF family gene, involved in functioning of blood vessels muscle cells). Thickening and fibrosis of small and medium sized cerebral vessels is present leading to recurrent ischemic strokes, migraine, dementia and behavioural changes.

\section{CARASIL (Cerebral Autosomal Recessive Arteriop- athy with Subcortical Infarcts and Leukoencepha- lopathy)}

It is inherited in AR fashion. Thickening and fibrosis of small sized cerebral vessels is present resulting in ischemic strokes, patchy alopecia and spondyloarthropathies.

\section{Sneddon's syndrome}

A rare non-inflammatory thrombotic vasculopathy characterized by the combination of cerebrovascular disease with livedo racemose (LR). LR is defined as a dusky erythematous to-violaceous, irregular, net-like pattern in the skin. LR may precede the onset of stroke by years and the trunk and/or buttocks are involved in nearly all patients. The cerebrovascular manifestations are mostly secondary to ischemia [8]. Histology may reveal thrombosis of subcutaneous arterioles and compensatory capillary dilation with blood stagnation causing LR [8]. Vascular thrombosis and recanalization within skin and brain tissue support the pathophysiology of coagulopathy. The cerebrovascular manifestations are mostly secondary to ischemia.

\section{Hereditary hemorrhagic telangiectasia (Osler we- ber rendu syndrome)}

$A D$ inheritance pattern with abnormal blood vessel formation in skin, mucous membranes, solid organs like lungs, liver or brain. Leads to ICH and SAH in some cases.

\section{Retinal Vasculopathy and Cerebral Leukodystro- phy (RVCL)}

Hereditary endotheliopathy with retinopathy, nephropathy and stroke (HERNS), cerebroretinal vasculopathy (CRV) and hereditary vascular retinopathy (HVR) are conditions which have common etiology. They are phenotypic variants of the monogenic autosomal dominant inherited entity-retinal vasculopathy and cerebral leukodystrophy (RVCL) [9]. They are caused by mutation in TREX1(Three prime Repair EXonuclease) gene located on chromosome 3 which encode autonomous DNA nucleases responsible for genome stability. A pathological study shows a vasculopathy with multi-laminated vascular basement membrane in brain and other organs (kidneys, stomach and intestines) [9]. 


\section{SUSAC'S syndrome}

An immune-mediated, pauci-inflammatory, ischemia-producing, occlusive microvascular endotheliopathy/basement membranopathy that affects the brain, retina, and inner ear. Though the exact pathogenesis of this disorder is unknown, the retinal and brain biopsy findings suggest a small vessel vasculopathy leading to arteriolar occlusion and microinfarction of cerebral, retinal and cochlear tissue [10].

\section{Metabolic Disorders}

\section{Fabry disease}

An x-linked lysosomal storage disease, due to deficiency of a lysosomal hydrolase (alpha galactosidase A). Accumulation of GAGs (glycotriosylceramide) in lysosomes of cells including vascular endothelium leads to vessel occlusion and strokes [11]. It can affect both small and large vessels.

\section{Homocystinuria}

It is inherited in AR fashion and occurs due to defects in methionine metabolism. Deficiency of cystathionine B-synthase that converts homocysteine to cystathionine leads to elevated serum and urine homocysteine. Elevated homocysteine causes endothelial dysfunction and promotes endothelial smooth muscle proliferation with resultant atherosclerosis leading to thrombosis and stroke.

\section{MELAS (Mitochondrial Encephalomyopathy Lactic Acidosis and Stroke like Episodes)}

A metabolic, multisystem and progressive neurodegenerative disorder. Several mutations have been described which affect mitochondrial metabolism. It is common in people aged 4-15 years [12]. Due to transient oxidative phosphorylation disorder within the brain cells, free radical production is enhanced leading to vasoconstriction and alterations in Nitric oxide homeostasis. It can also occur in infancy and cause developmental delays.

\section{Pompe's disease}

Pompe disease is a rare (estimated at 1 in every 40,000 births [13]. inherited and often fatal disorder that disables the heart and skeletal muscles. It is caused by mutations in a gene that code for acid alpha-glucosidase (GAA). GAA breaks down glycogen in lysosomes. In Pompe disease, mutations in the GAA gene reduce or completely eliminate this essential enzyme. Excessive amounts of lysosomal glycogen accumulate everywhere in the body, but the cells of the heart and skeletal muscles are the most seriously affected. Researchers have identified up to 300 different mutations in the GAA gene that cause the symptoms of Pompe disease, which can vary widely in terms of age of onset and severity. The severity of the disease and the age of onset are related to the degree of enzyme deficiency.
While Pompe disease is mainly characterized by muscular weakness and respiratory and cardiac insufficiency, patients also may develop complications in vessels that supply blood to the brain [14]. These changes were previously reported to affect patients' long-term survival, as they increased the risk for intracranial hemorrhages, compression of cranial nerves, and stroke.

More recent studies have reported that the main blood vessel affected in the brain of Pompe disease patients is the basilar artery, which supplies oxygen-rich blood to some of the most critical parts of the brain. In Pompe patients, the artery can show signs of elongation, dilatation, and/or tortuosity, a condition known as dolichoectasia [15].

Vasculopathy is an under recognized complication of Pompe disease. The subsequent intracerebral and subarachnoid hemorrhage can be the presenting feature leading to diagnosis of Pompe disease, despite symptoms of the disease often being present for a number of years previously [13].

Dolichoectasia of intracranial vessels in cases with stroke in young, with or without muscle weakness, should raise a differential of late onset Pompe's disease [15].

\section{Cerebrotendinous xanthomatosis}

It is inherited in AD fashion. A defect in CYP27A1 gene (produces an enzyme that degrades cholesterol) leads to inability to metabolize cholesterol properly [16]. Cholesterol deposits in tissues (xanthomas), but blood cholesterol levels are normal. Xanthomas in brain cause progressive neurological damage (dementia, seizures) and can obstruct brain blood supply leading to stroke.

\section{Connective Tissue Disorders}

\section{Marfan syndrome}

It is inherited in AD pattern. Disease occurs due to mutations in fibrillin 1 , an ECM protein. Connection between elastic fibers and smooth muscle cells are defective. It is characterized by elastic fiber calcification, intimal hyperplasia and structural collapse of vessel wall. Aortic incompetence and dissection are commoner than strokes.

\section{Ether-Danlos syndrome}

$A D$ pattern with mutation in gene for type 3 collagen. Collagen formed has reduced strength and elasticity. There is presence of intra-cerebral aneurysms and aortic aneurysms. If aneurysmal rupture occurs, it will result in a stroke.

\section{Drugs \\ Cocaine}

It causes acute hypertension and drug induced vasculopathy. 


\section{Oral contraceptives}

It causes hyper-viscosity of blood due to its estrogen component.

\section{Miscellaneous}

\section{Infective endocarditis}

Vegetations can lead to cardio-embolic stroke.

\section{Post myocardial infarction}

Akinetic and dyskinetic portions of affected myocardium encourage formation of intra-cardiac thrombi which can embolize to cerebral vessels. Risk is greatest within the first 2 weeks following a myocardial infarction.

\section{Congenital heart defects}

Paradoxical emboli from patent foramen ovale cause strokes instead of pulmonary embolism [1].

\section{Migrainous stroke}

It commonly occurs with aura.

\section{Trauma}

It can cause shearing of intra-cerebral blood vessels and lead to a stroke.

\section{Management}

This requires proper clinical and laboratory evaluation.

Imaging is important to visualize site of lesion, blood and urine tests are necessary to identify specific cause while genetic tests are important for hereditary diseases.

\section{Laboratory investigations for specific diseases}

Cardiac embolism : Echocardiography (including transesophageal)

Premature atherosclerosis : Serum lipids

Arterial dissection : MRI, Angiography

Thrombophilia : Protein C, protein S, Antithrombin III, Factor V Leiden,

prothrombin time, Platelet count

Homocystinuria : Urinary amino acids, Methionine loading test

Antiphospholipid antibody : Anticardiolipin antibodies/lupus anticoagulant syndrome

SLE : Antinuclear antibodies

Vasculitis : ESR, CRP, Antineutrophil cytoplasmic antibody (ANCA)

CADASIL : MRI brain, Genetic analysis, Skin biopsy

Mitochondrial cytopathy : Serum lactate, White cell mitochondrial DNA, Muscle biopsy,

Mitochondrial molecular genetics

Fabry's disease : Alpha-galactosidase levels in blood

Arteriovenous malformation : MRI/angiography

Drug misuse : Drug screen (amphetamine, cocaine)

Coagulopathy : Prothrombin time (PT) and activated, partial thromboplastin time

(APTT)

\section{Treatment}

Usually requires a multi-disciplinary input. General principles of stroke management also apply. Non-pharmacological therapy involves counselling and support in disorders like Marfan, Ehlers-Danlos, CARDASIL and CARASIL, which are life-long and have no cure currently available. Dietary modification is appropriate in MELAS [12], where arginine supplementation is recommended and in homocystinuria where methionine restriction is encouraged.

Pharmacological therapy involves the use of anticoagulation in thrombophilic disorders e.g. Factor $\mathrm{V}$ Leiden Protein $C$ \& $S$ deficiency. Bulsphan reduces red blood cell sickling in SCD. Anti-neoplastic drugs are appropriate in myeloproliferative disorders e.g. PRV, ET. Patients using OCPs and cocaine are advised to stop.

Surgery interventions include vascular bypass surgeries in patients with Moyamoya Disease. AV aneurysmal clipping can be performed in selected cases of Polyarteritis nodosa [17].

\section{Conclusion}

Multiple rare and hereditary causes of stroke have been reviewed. Poor diagnostic facilities is a major limitation in Africa.

Stroke in the young is fetching attention on a global scale $[18,19]$. These rare causes should be looked for in cases where conventional risk factors are absent.

\section{Limitations}

No local studies or literature review could be found concerning hereditary causes of stroke.

\section{Declaration}

No conflict of interest is declared.

\section{References}

1. Francis J, Raghunathan S, Khanna $P$ (2007) The role of genetics in stroke. Postgrad Med J 83: 590-595.

2. Malik R, Chauhan G, Traylor M, Sargurupremraj M, Okada $Y$, et al. (2018) Multiancestry genome-wide association study of 520,000 subjects identifies 32 loci associated with stroke and stroke subtypes. Nature Genetics 50: 524-537.

3. Alberts MJ (2003) Genetics of cerebrovascular disease. Advances in Stroke 35: 342-344. 
4. Bucczek J, Czlonkowska A (2012) Stroke and genetics. Period Biolog 114: 259-266.

5. Markus H (2012) Stroke genetics: Prospects for personalized medicine. BMC Med 10: 113.

6. Griffiths D, Sturm J (2011) Epidemiology and etiology of young stroke. Stroke Res Treatment 3: 209-370.

7. Onwuchekwa AC, Onwuchekwa RC, Asekomeh E (2009) Stroke in young Nigerian adults. J Vasc Nurs 27: 98-102.

8. Shengjun Wu, Ziqi Xu, Hui Lian (2014) Sneddon's syndrome: A comprehensive review of the literature. Orphanet J Rare Dis 9: 215.

9. Richards A, Maagdenberg VD, Jen JC, Kavanagh D, Bertram P, et al. (2007) C- terminal truncation in human 3'5' DNA exonuclease TREX1 cause autosomal dominant vasculopathy with cerebral leukodystrophy. Nat Genet 39: 1068-1070.

10. Vicktoria Vishnevskia-Dai, Joav Chapman, Roee Sheinfeld, Tal Sharon, Ruth Huna-Baron, et al. (2016) Susac syndrome: Clinical characteristics, clinical classification, and long-term prognosis. Medicine (Baltimore) 95: e5223.

11. Grewal RP (1994) Stroke in Fabry's disease. J Neurol 241: 153-156.

12. Enter C, Muller-Hocker J, Zierz S, Kurlemann G, Pongratz D, et al. (1991) A specific point mutation in the mitochondrial ge- nome of Caucasians with MELAS. Hum Genet 88: 233-236.

13. James Lilleker, Mark Roberts, Bernard Boothman (2013) Dilative vasculopathy and Cerebral Haemorrhage as a presentation of late-onset Pompe disease. Association of British Neurologists $(A B N)$ joint meeting with the Royal College of Physicians (RCP), London.

14. Finsterer $\mathrm{J}$ (2012) Dilative arteriopathy in metabolic myopathies, particularly Pompe's disease. Acta Neurol Belg 112: 15-18.

15. Huded V, Bohra V, Prajapati J, DeSouza R, Ramankutty R (2016) Stroke in young-dilative arteriopathy: A clue to late-onset pompe's disease? J Stroke Cerebrovasc Dis 25: e50-e52.

16. Van den Berg JS, Hennekam RC, Cruysberg JR, Steijlen PM, Swart J, et al. (2000) Prevalence of symptomatic intracranial aneurysm and ischemic stroke in pseudoxanthoma elasticum. Cerebrovasc Dis 10: 315-319.

17. Marcoux M (2000) Stroke in young adults. CNI Rev Med J 11: 2 .

18. Zannad F, Visvikis S, Gueguen R, Sass C, Chapet O, et al. (1998) Genetics strongly determines the wall thickness of the left and right carotid arteries. Hum Genet 103: 183-188.

19. Jartti L, Rönnemaa T, Kaprio J, Järvisalo MJ, Toikka JO, et al. (2002) Population based twin study of the effects of migration from Finland to Sweden on endothelial function and intima-media thickness. Arterio Thrombo Vasc Biol 22: 832-837. 